\title{
Antisynchronization of Nonidentical Fractional-Order Chaotic Systems Using Active Control
}

\author{
Sachin Bhalekar ${ }^{1}$ and Varsha Daftardar-Gejji ${ }^{2}$ \\ ${ }^{1}$ Department of Mathematics, Shivaji University, Vidyanagar, Kolhapur 416004, India \\ ${ }^{2}$ Department of Mathematics, University of Pune, Ganeshkhind, Pune 411007, India \\ Correspondence should be addressed to Varsha Daftardar-Gejji, vsgejji@gmail.com
}

Received 7 May 2011; Accepted 16 July 2011

Academic Editor: Wen Chen

Copyright (C) 2011 S. Bhalekar and V. Daftardar-Gejji. This is an open access article distributed under the Creative Commons Attribution License, which permits unrestricted use, distribution, and reproduction in any medium, provided the original work is properly cited.

\begin{abstract}
Antisynchronization phenomena are studied in nonidentical fractional-order differential systems. The characteristic feature of antisynchronization is that the sum of relevant state-variables vanishes for sufficiently large value of time variable. Active control method is used first time in the literature to achieve antisynchronization between fractional-order Lorenz and Financial systems, Financial and Chen systems, and Lü and Financial systems. The stability analysis is carried out using classical results. We also provide numerical results to verify the effectiveness of the proposed theory.
\end{abstract}

\section{Introduction}

In their pioneering work [1, 2], Pecora and Carroll have shown that chaotic systems can be synchronized by introducing appropriate coupling. The notion of synchronization of chaos has further been explored in secure communications of analog and digital signals [3] and for developing safe and reliable cryptographic systems [4]. For the synchronization of chaotic systems, a variety of approaches have been proposed which include nonlinear feedback [5], adaptive [6,7], and active controls [8,9].

Antisynchronization (AS) is a phenomenon in which the state vectors of the synchronized systems have the same amplitude but opposite signs to those of the driving system. Hence the sum of two signals converges to zero when AS appears. Antisynchronization has applications in lasers [10], in periodic oscillators, and in communication systems. Using AS to lasers, one may generate not only drop-outs of the intensity but also short pulses of high intensity, which results in the pulses of special shapes. 
Active control method is used to AS for two identical integer order systems by Ho et al. [11] and for nonidentical systems by Li and Zhou [12]. Nonlinear control scheme was used by Li et al. [13] to study AS. Al-Sawalha [14] have reported AS between Chua's system and Nuclear spin generator (NSG) system. Recently AS between Lorenz system, Lü system, and Four-scroll system is investigated by Elabbasy and El-Dessoky [15].

Fractional calculus deals with derivatives and integration of arbitrary order [16-18] and has deep and natural connections with many fields of applied mathematics, engineering, and physics. Fractional calculus has a wide range of applications in control theory [19], viscoelasticity [20], diffusion [21-27], turbulence, electromagnetism, signal processing [28, 29], and bioengineering [30]. Analysis of fractional-order dynamical systems involving Riemann-Liouville as well as Caputo derivatives has been dealt with by present authors $[31,32]$.

Synchronization of fractional-order chaotic systems was first studied by Deng and Li [33] who carried out synchronization in case of the fractional Lü system. Further they have investigated synchronization of fractional Chen system [34]. Li and Deng have summarized the theory and techniques of synchronization in [35]. The theory for synchronization problems in an $\omega$-symmetrically coupled fractional differential systems have been studied by Zhou and Li [36]. Since then many fractional systems have been investigated by various researchers. A few examples in this regards are Li et al. [37] (Chua system), Wang et al. [38] (Chen system), Wang and Zhang [39] (unified system), Wang and He [40] (unified system), Yu and Li [41] (Rossler hyperchaos system), and Tavazoei and Haeri [42] (Lü system and Chen system). Of late Matouk [43] has synchronized fractional Lü system with fractional Chen system and fractional Chen system with fractional Lorenz system. Hu et al. [44] have synchronized fractional Lorenz and fractional Chen systems. Further Bhalekar and Daftardar-Gejji [45] have investigated the interrelationship between the (fractional) order and synchronization in different chaotic dynamical systems. However, it seems that there are no previous results on AS of two nonidentical fractional-order chaotic systems.

In the present paper, we study the antisynchronization of the following fractional systems using active control method: (i) Lorenz with Financial, (ii) Financial with Chen, and (iii) Lü with Financial.

\section{Preliminaries}

\subsection{Fractional Calculus}

Basic definitions and properties of fractional derivative/integrals are given below $[16,17,46]$.

Definition 2.1. A real function $f(t), t>0$, is said to be in space $C_{\alpha}, \alpha \in \mathfrak{R}$ if there exists a real number $p(>\alpha)$, such that $f(t)=t^{p} f_{1}(t)$ where $f_{1}(t) \in C[0, \infty)$.

Definition 2.2. A real function $f(t), t>0$, is said to be in space $C_{\alpha}^{m}, m \in \mathbb{N} \cup\{0\}$ if $f^{(m)} \in C_{\alpha}$.

Definition 2.3. Let $f \in C_{\alpha}$ and $\alpha \geq-1$, then the (left-sided) Riemann-Liouville integral of order $\mu, \mu>0$ is given by

$$
I^{\mu} f(t)=\frac{1}{\Gamma(\mu)} \int_{0}^{t}(t-\tau)^{\mu-1} f(\tau) d \tau, \quad t>0
$$


Definition 2.4. The (left-sided) Caputo fractional derivative of $f, f \in C_{-1}^{m}, m \in \mathbb{N} \cup\{0\}$, is defined as

$$
\begin{aligned}
D^{\mu} f(t) & =\frac{d^{m}}{d t^{m}} f(t), \quad \mu=m \\
& =I^{m-\mu} \frac{d^{m} f(t)}{d t^{m}}, \quad m-1<\mu<m, \quad m \in \mathbb{N} .
\end{aligned}
$$

Note that for $m-1<\mu \leq m, m \in \mathbb{N}$,

$$
\begin{gathered}
I^{\mu} D^{\mu} f(t)=f(t)-\sum_{k=0}^{m-1} \frac{d^{k} f}{d t^{k}}(0) \frac{t^{k}}{k !}, \\
I^{\mu} t^{v}=\frac{\Gamma(v+1)}{\Gamma(\mu+v+1)} t^{\mu+v} .
\end{gathered}
$$

\subsection{Numerical Method for Solving Fractional Differential Equations}

Numerical methods used for solving ODEs have to be modified for solving fractional differential equations (FDEs). A modification of Adams-Bashforth-Moulton algorithm is proposed by Diethelm et al. in [47-49] to solve FDEs.

Consider for $\alpha \in(m-1, m]$ the initial value problem (IVP)

$$
\begin{gathered}
D^{\alpha} y(t)=f(t, y(t)), \quad 0 \leq t \leq T, \\
y^{(k)}(0)=y_{0}^{(k)}, \quad k=0,1, \ldots, m-1 .
\end{gathered}
$$

The IVP (2.4) is equivalent to the Volterra integral equation

$$
y(t)=\sum_{k=0}^{m-1} y_{0}^{(k)} \frac{t^{k}}{k !}+\frac{1}{\Gamma(\alpha)} \int_{0}^{t}(t-\tau)^{\alpha-1} f(\tau, y(\tau)) d \tau
$$

Consider the uniform grid $\left\{t_{n}=n h / n=0,1, \ldots, N\right\}$ for some integer $N$ and $h:=T / N$. Let $y_{h}\left(t_{n}\right)$ be approximation to $y\left(t_{n}\right)$. Assume that we have already calculated approximations $y_{h}\left(t_{j}\right), j=1,2, \ldots, n$, and we want to obtain $y_{h}\left(t_{n+1}\right)$ by means of the equation

$$
y_{h}\left(t_{n+1}\right)=\sum_{k=0}^{m-1} \frac{t_{n+1}^{k}}{k !} y_{0}^{(k)}+\frac{h^{\alpha}}{\Gamma(\alpha+2)} f\left(t_{n+1}, y_{h}^{P}\left(t_{n+1}\right)\right)+\frac{h^{\alpha}}{\Gamma(\alpha+2)} \sum_{j=0}^{n} a_{j, n+1} f\left(t_{j}, y_{n}\left(t_{j}\right)\right),
$$

where

$$
a_{j, n+1}= \begin{cases}n^{\alpha+1}-(n-\alpha)(n+1)^{\alpha} & \text { if } j=0 \\ (n-j+2)^{\alpha+1}+(n-j)^{\alpha+1}-2(n-j+1)^{\alpha+1} & \text { if } 1 \leq j \leq n \\ 1 & \text { if } j=n+1 .\end{cases}
$$


The preliminary approximation $y_{h}^{P}\left(t_{n+1}\right)$ is called predictor and is given by

$$
y_{h}^{P}\left(t_{n+1}\right)=\sum_{k=0}^{m-1} \frac{t_{n+1}^{k}}{k !} y_{0}^{(k)}+\frac{1}{\Gamma(\alpha)} \sum_{j=0}^{n} b_{j, n+1} f\left(t_{j}, y_{n}\left(t_{j}\right)\right)
$$

where

$$
b_{j, n+1}=\frac{h^{\alpha}}{\alpha}\left((n+1-j)^{\alpha}-(n-j)^{\alpha}\right) .
$$

Error in this method is

$$
\max _{j=0,1, \ldots, N}\left|y\left(t_{j}\right)-y_{h}\left(t_{j}\right)\right|=O\left(h^{p}\right)
$$

where $p=\min (2,1+\alpha)$.

\section{System Description}

The fractional-order Lorenz system $[50,51]$ is described by

$$
\begin{gathered}
D^{\alpha} x=\sigma(y-x), \\
D^{\alpha} y=r x-y-x z, \\
D^{\alpha} z=x y-\mu z,
\end{gathered}
$$

where $\sigma=10$ is the Prandtl number, $r=28$ is the Rayleigh number over the critical Rayleigh number, and $\mu=8 / 3$ gives the size of the region approximated by the system. The minimum effective dimension for this system is 2.97 [51].

In [52] Chen proposed the financial system to fractional-order

$$
\begin{aligned}
& D^{\alpha} x=z+(y-a) x \\
& D^{\alpha} y=1-b y-x^{2} \\
& D^{\alpha} z=-x-c z
\end{aligned}
$$

where $a=3, b=0.1$, and $c=1$. The minimum effective dimension for which the system exhibits chaos is given by 2.32 [52].

Li and Peng [53] studied chaos in fractional-order Chen system

$$
\begin{aligned}
& D^{\alpha} x=a_{1}(y-x) \\
& D^{\alpha} y=\left(c_{1}-a_{1}\right) x-x z+c_{1} y \\
& D^{\alpha} z=x y-b_{1} z
\end{aligned}
$$

where $a_{1}=35, b_{1}=3$, and $c_{1}=27$. The minimum effective dimension reported is 2.92 [53]. 
Fractional-order Lü system is the lowest-order chaotic system among all the chaotic systems reported in the literature [54]. The minimum effective dimension reported is 0.30 . The system is given by

$$
\begin{aligned}
& D^{\alpha} x=a_{2}(y-x), \\
& D^{\alpha} y=c_{2} y-x z, \\
& D^{\alpha} z=x y-b_{2} z,
\end{aligned}
$$

where $a_{2}=35, b_{2}=3$, and $c_{2}=28$.

\section{Antisynchronization between Fractional-Order Lorenz and Financial System}

In this section, we study the antisynchronization between Lorenz and Financial systems. Assuming that the Lorenz system drives the Financial system, we define the drive (master) and response (slave) systems as follows:

$$
\begin{aligned}
& D^{\alpha} x_{1}=\sigma\left(y_{1}-x_{1}\right) \\
& D^{\alpha} y_{1}=r x_{1}-y_{1}-x_{1} z_{1} \\
& D^{\alpha} z_{1}=x_{1} y_{1}-\mu z_{1} \\
& D^{\alpha} x_{2}=z_{2}+\left(y_{2}-a\right) x_{2}+u_{1}(t), \\
& D^{\alpha} y_{2}=1-b y_{2}-x_{2}^{2}+u_{2}(t) \\
& D^{\alpha} z_{2}=-x_{2}-c z_{2}+u_{3}(t)
\end{aligned}
$$

The unknown terms $u_{1}, u_{2}, u_{3}$ in (4.2) are active control functions to be determined. Define the error functions as

$$
e_{1}=x_{1}+x_{2}, \quad e_{2}=y_{1}+y_{2}, \quad e_{3}=z_{1}+z_{2} .
$$

Equation (4.3) together with (4.1) and (4.2) yields the error system

$$
\begin{aligned}
& D^{\alpha} e_{1}=(a-\sigma) x_{1}+\sigma y_{1}+x_{1} y_{1}-z_{1}-a e_{1}-y_{1} e_{1}-x_{1} e_{2}+e_{1} e_{2}+e_{3}+u_{1}(t), \\
& D^{\alpha} e_{2}=1+r x_{1}-x_{1}^{2}+(b-1) y_{1}-x_{1} z_{1}+2 x_{1} e_{1}-e_{1}^{2}-b e_{2}+u_{2}(t), \\
& D^{\alpha} e_{3}=x_{1}+(c-\mu) z_{1}+x_{1} y_{1}-e_{1}-c e_{3}+u_{3}(t) .
\end{aligned}
$$


We define active control functions $u_{i}(t)$ as

$$
\begin{aligned}
& u_{1}(t)=V_{1}(t)-(a-\sigma) x_{1}-\sigma y_{1}-x_{1} y_{1}+z_{1}+y_{1} e_{1}+x_{1} e_{2}-e_{1} e_{2} \\
& u_{2}(t)=V_{2}(t)-1-r x_{1}+x_{1}^{2}-(b-1) y_{1}+x_{1} z_{1}-2 x_{1} e_{1}+e_{1}^{2} \\
& u_{3}(t)=V_{3}(t)-x_{1}-(c-\mu) z_{1}-x_{1} y_{1}
\end{aligned}
$$

The terms $V_{i}(t)$ are linear functions of the error terms $e_{i}(t)$. With the choice of $u_{i}(t)$ given by (4.5), the error system (4.5) becomes

$$
\begin{aligned}
& D^{\alpha} e_{1}=-a e_{1}-e_{3}+V_{1}(t), \\
& D^{\alpha} e_{2}=-b e_{2}+V_{2}(t), \\
& D^{\alpha} e_{3}=-e_{1}-c e_{3}+V_{3}(t) .
\end{aligned}
$$

The control terms $V_{i}(t)$ are chosen so that the system (4.6) becomes stable. There is not a unique choice for such functions. We choose

$$
\left(\begin{array}{l}
V_{1} \\
V_{2} \\
V_{3}
\end{array}\right)=A\left(\begin{array}{l}
e_{1} \\
e_{2} \\
e_{3}
\end{array}\right)
$$

where $A$ is a $3 \times 3$ real matrix, chosen so that for all eigenvalues $\lambda_{i}$ of the system (4.6) the condition

$$
\left|\arg \left(\lambda_{i}\right)\right|>\frac{\alpha \pi}{2}
$$

is satisfied. (The stability condition (4.8) is discussed in the literature [55-57]). If we choose

$$
A=\left(\begin{array}{ccc}
a-1 & 0 & -1 \\
0 & -1+b & 0 \\
1 & 0 & c-1
\end{array}\right)
$$

then the eigenvalues of the linear system (4.6) are $-1,-1$, and -1 . Hence the condition (4.8) is satisfied for $\alpha<2$. Since we consider only the values $\alpha \leq 1$, we get the required antisynchronization.

\subsection{Simulation and Results}

Parameters of the Lorenz system are taken as $\sigma=10, r=28, \mu=8 / 3$ and Financial system as $a=3, b=0.1, c=1$. The fractional-order $\alpha$ is taken to be 0.99 for which both the systems are chaotic. The initial conditions for drive and response system are $x_{1}(0)=10, y_{1}(0)=5$, 


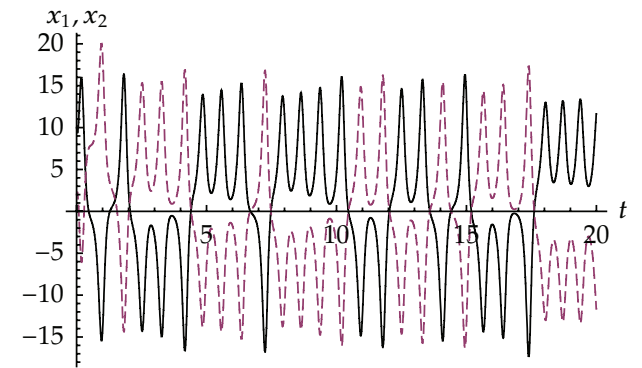

(a)

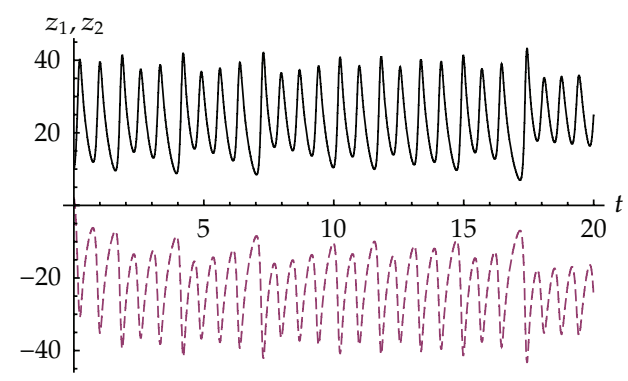

(c)

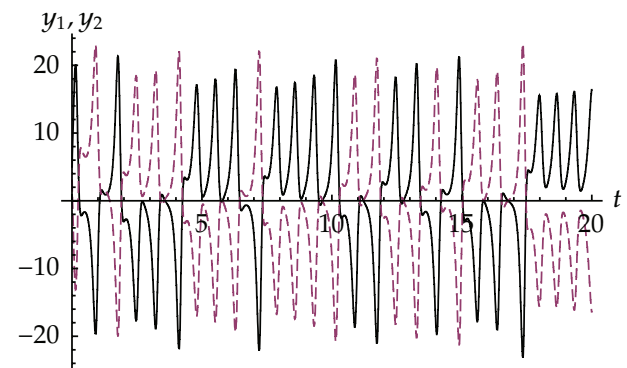

(b)

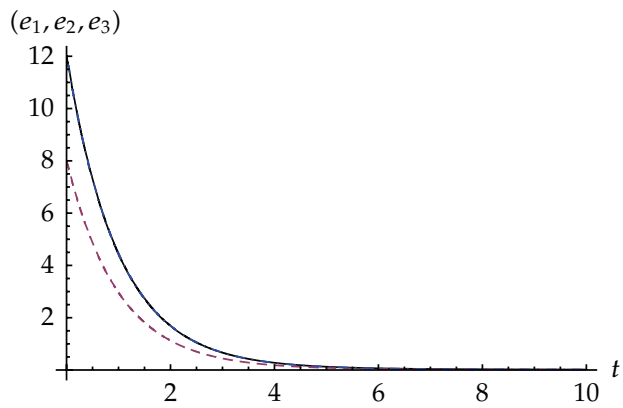

(d)

Figure 1: (a) Signals $x_{1}, x_{2}$, (b) Signals $y_{1}, y_{2}$, (c) Signals $z_{1}, z_{2}$, and (d) Error system.

$z_{1}(0)=10$ and $x_{2}(0)=2, y_{2}(0)=3, z_{2}(0)=2$, respectively. Initial conditions for the error system are thus $e_{1}(0)=12, e_{2}(0)=8$, and $e_{3}(0)=12$. Figures $1(\mathrm{a})-1(\mathrm{c})$ show the antisynchronization between Lorenz and Financial system; the response system is given by dashed line. The errors $e_{1}(t)$ (solid line), $e_{2}(t)$ (dashed line) and $e_{3}(t)$ (dot-dashed line) in the antisynchronization are shown in Figure 1(d).

\section{Antisynchronization between Financial and Chen Systems of Fractional Order}

Assuming that Chen system is antisynchronized with Financial system; define the drive system as

$$
\begin{aligned}
& D^{\alpha} x_{1}=z_{1}+\left(y_{1}-a\right) x_{1}, \\
& D^{\alpha} y_{1}=1-b y_{1}-x_{1}^{2}, \\
& D^{\alpha} z_{1}=-x_{1}-c z_{1}
\end{aligned}
$$

and the response system as

$$
\begin{aligned}
& D^{\alpha} x_{2}=a_{1}\left(y_{2}-x_{2}\right)+u_{4} \\
& D^{\alpha} y_{2}=\left(c_{1}-a_{1}\right) x_{2}-x_{2} z_{2}+c_{1} y_{2}+u_{5}, \\
& D^{\alpha} z_{2}=x_{2} y_{2}-b_{1} z_{2}+u_{6} .
\end{aligned}
$$


Let $e_{1}=x_{1}+x_{2}, e_{2}=y_{1}+y_{2}$, and $e_{3}=z_{1}+z_{2}$ be error functions. For antisynchronization, it is essential that the errors $e_{i} \rightarrow 0$ as $t \rightarrow \infty$. Note that

$$
\begin{aligned}
D^{\alpha} e_{1}= & \left(a_{1}-a\right) x_{1}-a_{1} y_{1}+x_{1} y_{1}+z_{1}-a_{1} e_{1}+a_{1} e_{2}+u_{4}(t), \\
D^{\alpha} e_{2}= & 1+\left(a_{1}-c_{1}\right) x_{1}-x_{1}^{2}-\left(b+c_{1}\right) y_{1}-x_{1} z_{1} \\
& +\left(c_{1}-a_{1}\right) e_{1}+z_{1} e_{1}+c_{1} e_{2}+x_{1} e_{3}-e_{1} e_{3}+u_{5}(t), \\
D^{\alpha} e_{3}= & -x_{1}+x_{1} y_{1}+\left(b_{1}-c\right) z_{1}-y_{1} e_{1}-x_{1} e_{2}+e_{1} e_{2}-b_{1} e_{3}+u_{6}(t) .
\end{aligned}
$$

The control functions are chosen as

$$
\begin{aligned}
& u_{4}=V_{4}-\left(a_{1}-a\right) x_{1}+a_{1} y_{1}-x_{1} y_{1}-z_{1} \\
& u_{5}=V_{5}-1-\left(a_{1}-c_{1}\right) x_{1}+x_{1}^{2}+\left(b+c_{1}\right) y_{1}+x_{1} z_{1}-z_{1} e_{1}-x_{1} e_{3}+e_{1} e_{3} \\
& u_{6}=V_{6}+x_{1}-x_{1} y_{1}-\left(b_{1}-c\right) z_{1}+y_{1} e_{1}+x_{1} e_{2}-e_{1} e_{2} .
\end{aligned}
$$

The linear functions $V_{4}, V_{5}, V_{6}$ are given by

$$
\begin{aligned}
& V_{4}=\left(a_{1}-1\right) e_{1}-a_{1} e_{2}, \\
& V_{5}=-\left(a_{1}-c_{1}\right) e_{1}-\left(c_{1}+1\right) e_{2}, \\
& V_{6}=\left(b_{1}-1\right) e_{3} .
\end{aligned}
$$

With the values given in (5.4) and (5.5), the error system (5.3) becomes

$$
\left(\begin{array}{l}
D^{\alpha} e_{1} \\
D^{\alpha} e_{2} \\
D^{\alpha} e_{3}
\end{array}\right)=\left(\begin{array}{ccc}
-1 & 0 & 0 \\
0 & -1 & 0 \\
0 & 0 & -1
\end{array}\right)\left(\begin{array}{l}
e_{1} \\
e_{2} \\
e_{3}
\end{array}\right) .
$$

It can be observed that the coefficient matrix of the error system (5.6) has eigenvalues -1 , $-1,-1$. So the system is stable and antisynchronization is achieved.

\subsection{Simulations and Results}

We take parameters for fractional-order Chen system as $a_{1}=35, b_{1}=3, c_{1}=27$. Parameters for the Financial system are same as given in Section 4.1. Experiments are done for fixed value of fractional-order $\alpha=0.95$, which is same for drive and response system (5.1) and (5.2). The initial conditions for the systems (5.1) and (5.2) are $x_{1}(0)=2, y_{1}(0)=3, z_{1}(0)=2$ and $x_{2}(0)=10, y_{2}(0)=25, z_{2}(0)=36$, respectively. For the error system (5.6), the initial conditions turns out to be $e_{1}(0)=12, e_{2}(0)=28, e_{3}(0)=38$. The simulation results are summarized in Figure 2. Antisynchronization between fractional Financial and Chen system is shown in Figure 2(a) (signals $x_{1}, x_{2}$ ), Figure 2(b) (signals $y_{1}, y_{2}$ ), and Figure 2(c) (signals $\left.z_{1}, z_{2}\right)$. Note that the drive systems are shown by solid lines, whereas response systems are 


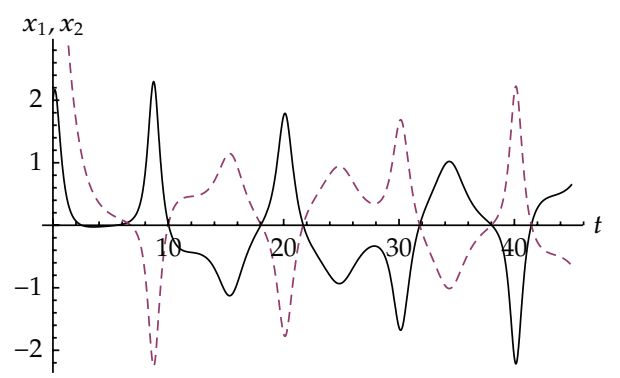

(a)

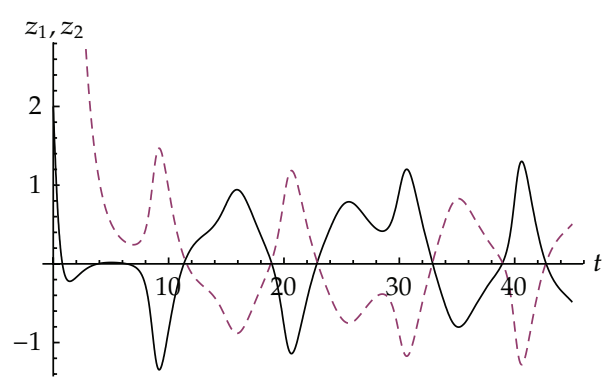

(c)

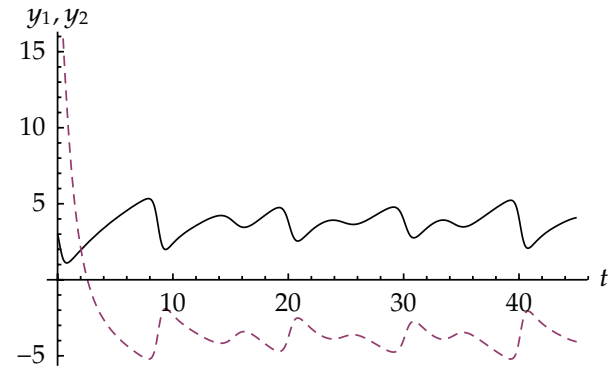

(b)

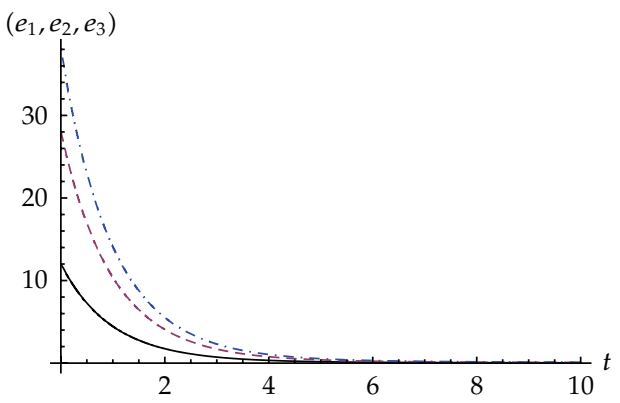

(d)

Figure 2: (a) Signals $x_{1}, x_{2}$, (b) Signals $y_{1}, y_{2}$, (c) Signals $z_{1}, z_{2}$, and (d) Error system.

shown by dashed lines. The errors $e_{1}(t)$ (solid line), $e_{2}(t)$ (dashed line), and $e_{3}(t)$ (dot-dashed line) in the antisynchronization are shown in Figure 2(d).

\section{Antisynchronization between Fractional Lü and Financial System}

In this case, consider Lü system as the drive system

$$
\begin{aligned}
& D^{\alpha} x_{1}=a_{2}\left(y_{1}-x_{1}\right), \\
& D^{\alpha} y_{1}=c_{2} y_{1}-x_{1} z_{1}, \\
& D^{\alpha} z_{1}=x_{1} y_{1}-b_{2} z_{1}
\end{aligned}
$$

and the response system as the Financial system

$$
\begin{aligned}
& D^{\alpha} x_{2}=z_{2}+\left(y_{2}-a\right) x_{2}+u_{7} \\
& D^{\alpha} y_{2}=1-b y_{2}-x_{2}^{2}+u_{8} \\
& D^{\alpha} z_{2}=-x_{2}-c z_{2}+u_{9}
\end{aligned}
$$


Let $e_{1}=x_{1}+x_{2}, e_{2}=y_{1}+y_{2}$, and $e_{3}=z_{1}+z_{2}$ be error functions. For antisynchronization, it is essential that the errors $e_{i} \rightarrow 0$ as $t \rightarrow \infty$. To achieve this one should choose the control terms $u_{7}, u_{8}, u_{9}$ properly. The error system thus becomes

$$
\begin{aligned}
& D^{\alpha} e_{1}=\left(a-a_{2}\right) x_{1}+a_{2} y_{1}+x_{1} y_{1}-z_{1}-a e_{1}-y_{1} e_{1}-x_{1} e_{2}+e_{1} e_{2}+e_{3}+u_{7}, \\
& D^{\alpha} e_{2}=1-x_{1}^{2}+\left(b+c_{2}\right) y_{1}-x_{1} z_{1}+2 x_{1} e_{1}-e_{1}^{2}-b e_{2}+u_{8} \\
& D^{\alpha} e_{3}=x_{1}+x_{1} y_{1}+\left(c-b_{2}\right) z_{1}-e_{1}-c e_{3}+u_{9} .
\end{aligned}
$$

The control functions are chosen as

$$
\begin{aligned}
& u_{7}=V_{7}-\left(a-a_{2}\right) x_{1}-a_{2} y_{1}-x_{1} y_{1}+z_{1}+y_{1} e_{1}+x_{1} e_{2}-e_{1} e_{2}, \\
& u_{8}=V_{8}-1+x_{1}^{2}-\left(b+c_{2}\right) y_{1}+x_{1} z_{1}-2 x_{1} e_{1}+e_{1}^{2}, \\
& u_{9}=V_{9}-x_{1}-x_{1} y_{1}-\left(c-b_{2}\right) z_{1} .
\end{aligned}
$$

The linear functions $V_{7}, V_{8}, V_{9}$ are given by

$$
\begin{aligned}
& V_{7}=(a-1) e_{1}-e_{3}, \\
& V_{8}=(-1+b) e_{2}, \\
& V_{9}=e_{1}+(c-1) e_{3} .
\end{aligned}
$$

With the values given in (6.4) and (6.5), the error system (6.3) becomes

$$
\left(\begin{array}{l}
D^{\alpha} e_{1} \\
D^{\alpha} e_{2} \\
D^{\alpha} e_{3}
\end{array}\right)=\left(\begin{array}{ccc}
-1 & 0 & 0 \\
0 & -1 & 0 \\
0 & 0 & -1
\end{array}\right)\left(\begin{array}{l}
e_{1} \\
e_{2} \\
e_{3}
\end{array}\right) .
$$

It can be observed that the coefficient matrix of the error system (6.6) has eigenvalues -1 , $-1,-1$. So the system is stable and antisynchronization is achieved.

\subsection{Simulations and Results}

Parameters for the Lü system are $a_{2}=35, b_{2}=3, c_{2}=28$, whereas parameters for Financial system are unaltered. The initial conditions for drive system are $x_{1}(0)=0.2, y_{1}(0)=0$, $z_{1}(0)=0.5$, whereas the initial conditions for response system are $x_{2}(0)=2, y_{2}(0)=3$, $z_{2}(0)=2$. Hence the initial conditions for the error system $(6.6)$ are $e_{1}(0)=2.2, e_{2}(0)=3$, $e_{3}(0)=2.5$. We perform the numerical simulations for fractional order $\alpha$, namely, 0.91 of the drive system (6.1) and response system (6.2). Figures 3(a), 3(b), and 3(c) show antisynchronization between fractional Lü and Financial system for $\alpha=0.91$. Figure 3(d) shows the errors $e_{1}(t)$ (solid line), $e_{2}(t)$ (dashed line), and $e_{3}(t)$ (dot-dashed line) in the antisynchronization for $\alpha=0.91$.

Mathematica 7 has been used for computations in the present paper. 


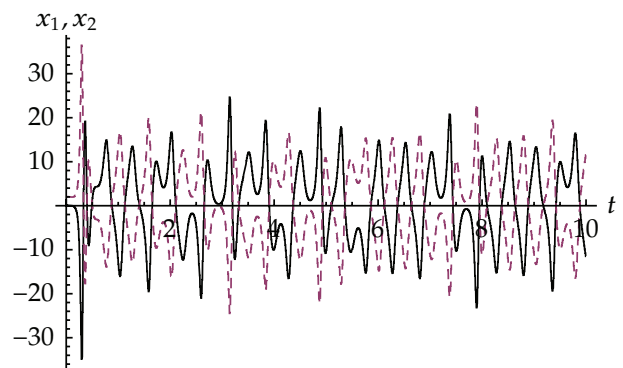

(a)

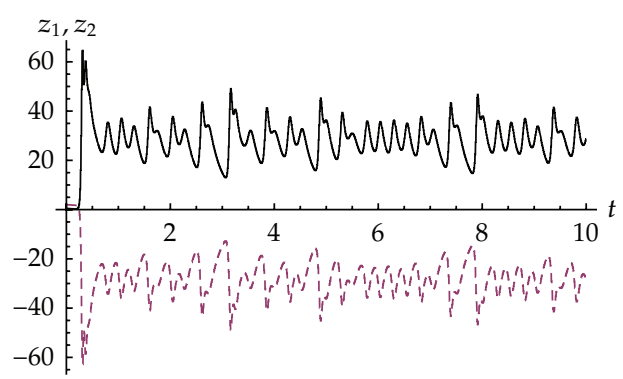

(c)

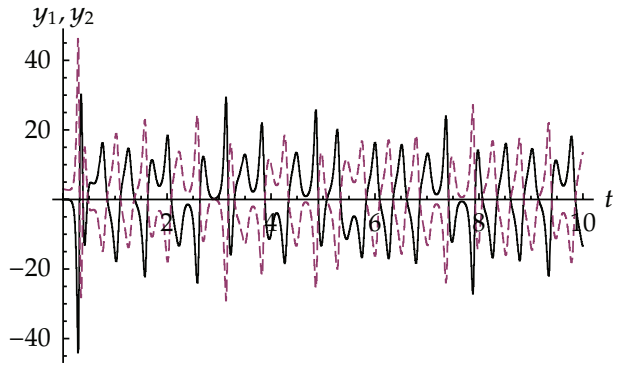

(b)

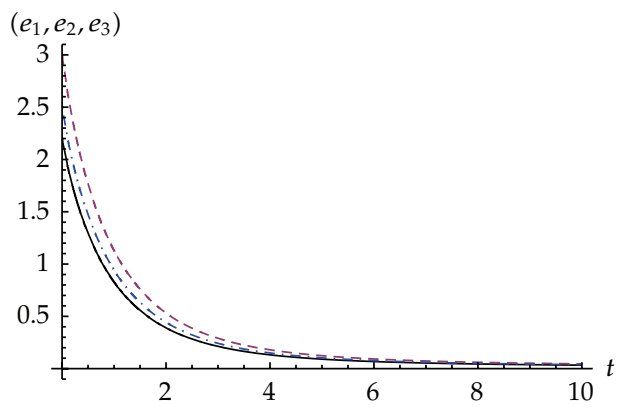

(d)

Figure 3: (a) $\alpha=0.91$, Signals $x_{1}, x_{2}$, (b) $\alpha=0.91$, Signals $y_{1}, y_{2}$, (c) $\alpha=0.91$, Signals $z_{1}, z_{2}$, and (d) $\alpha=0.91$, Error system.

\section{Conclusions}

Antisynchronization of nonidentical fractional-order chaotic systems has been done first time in the literature using active control. The fractional Financial system is controlled by fractional Lorenz system, the fractional Chen system is controlled by fractional Financial system, and the fractional Financial system is controlled by fractional Lü system.

\section{Acknowledgment}

V. Daftardar-Gejji acknowledges the Department of Science and Technology, N. Delhi, India for the Research Grants (project no. SR/S2/HEP-024/2009).

\section{References}

[1] L. M. Pecora and T. L. Carroll, "Synchronization in chaotic systems," Physical Review Letters, vol. 64, no. 8, pp. 821-824, 1990.

[2] L. M. Pecora and T. L. Carroll, "Driving systems with chaotic signals," Physical Review A, vol. 44, no. 4, pp. 2374-2383, 1991.

[3] R. Hilfer, Applications of Fractional Calculus in Physics, World Scientific Publishing, River Edge, NJ, USA, 2001.

[4] R. He and P. G. Vaidya, "Implementation of chaotic cryptography with chaotic synchronization," Physical Review E, vol. 57, no. 2, pp. 1532-1535, 1998.

[5] L. Huang, R. Feng, and M. Wang, "Synchronization of chaotic systems via nonlinear control," Physics Letters, Section A, vol. 320, no. 4, pp. 271-275, 2004. 
[6] T. L. Liao, "Adaptive synchronization of two Lorenz systems," Chaos, Solitons and Fractals, vol. 9, no. 9, pp. 1555-1561, 1998.

[7] M. T. Yassen, "Adaptive control and synchronization of a modified Chua's circuit system," Applied Mathematics and Computation, vol. 135, no. 1, pp. 113-128, 2003.

[8] E. W. Bai and K. E. Lonngren, "Synchronization of two Lorenz systems using active control," Chaos, Solitons and Fractals, vol. 8, no. 1, pp. 51-58, 1997.

[9] E. W. Bai and K. E. Lonngren, "Sequential synchronization of two Lorenz systems using active control," Chaos, Solitons and Fractals, vol. 11, no. 7, pp. 1041-1044, 2000.

[10] I. Wedekind and U. Parlitz, "Experimental observation of synchronization and anti-synchronization of chaotic low-frequency-fluctuations in external cavity semiconductor lasers," International Journal of Bifurcation and Chaos in Applied Sciences and Engineering, vol. 11, no. 4, pp. 1141-1147, 2001.

[11] M. C. Ho, Y. C. Hung, and C. H. Chou, "Phase and anti-phase synchronization of two chaotic systems by using active control," Physics Letters, Section A, vol. 296, no. 1, pp. 43-48, 2002.

[12] G. H. Li and S. P. Zhou, "Anti-synchronization in different chaotic systems," Chaos, Solitons and Fractals, vol. 32, no. 2, pp. 516-520, 2007.

[13] W. Li, X. Chen, and S. Zhiping, "Anti-synchronization of two different chaotic systems," Physica A, vol. 387, no. 14, pp. 3747-3750, 2008.

[14] A. Al-Sawalha, "Chaos anti-synchronization of two non-identical chaotic systems with known or fully unknown parameters," Chaos, Solitons and Fractals, vol. 42, no. 3, pp. 1926-1932, 2009.

[15] E. M. Elabbasy and M. M. El-Dessoky, "Adaptive anti-synchronization of different chaotic dynamical systems," Chaos, Solitons and Fractals, vol. 42, no. 4, pp. 2174-2180, 2009.

[16] I. Podlubny, Fractional Differential Equations, Academic Press, San Diego, Calif, USA, 1999.

[17] S. G. Samko, A. A. Kilbas, and O. I. Marichev, Fractional Integrals and Derivatives: Theory and Applications, Gordon and Breach Science, Yverdon, Switzerland, 1993.

[18] A. A. Kilbas, H. M. Srivastava, and J. J. Trujillo, Theory and Applications of Fractional Differential Equations, Elsevier, Amsterdam, The Netherlands, 2006.

[19] J. Sabatier, S. Poullain, P. Latteux, J. L. Thomas, and A. Oustaloup, "Robust speed control of a low damped electromechanical system based on CRONE control: application to a four mass experimental test bench," Nonlinear Dynamics, vol. 38, no. 1-4, pp. 383-400, 2004.

[20] M. Caputo and F. Mainardi, "A new dissipation model based on memory mechanism," Pure and Applied Geophysics, vol. 91, no. 1, pp. 134-147, 1971

[21] F. Mainardi, Y. Luchko, and G. Pagnini, "The fundamental solution of the space-time fractional diffusion equation," Fractional Calculus and Applied Analysis, vol. 4, no. 2, pp. 153-192, 2001.

[22] O. P. Agrawal, "Solution for a fractional diffusion-wave equation defined in a bounded domain," Nonlinear Dynamics, vol. 29, no. 1-4, pp. 145-155, 2002.

[23] V. Daftardar-Gejji and H. Jafari, "Boundary value problems for fractional diffusion-wave equations," The Australian Journal of Mathematical Analysis and Applications, vol. 3, pp. 1-18, 2006.

[24] V. Daftardar-Gejji and S. Bhalekar, "Solving multi-term linear and non-linear diffusion-wave equations of fractional order by Adomian decomposition method," Applied Mathematics and Computation, vol. 202, no. 1, pp. 113-120, 2008.

[25] H. Sun, W. Chen, and Y. Chen, "Variable-order fractional differential operators in anomalous diffusion modeling," Physica A, vol. 388, no. 21, pp. 4586-4592, 2009.

[26] I. S. Jesus and J. A. Tenreiro MacHado, "Fractional control of heat diffusion systems," Nonlinear Dynamics, vol. 54, no. 3, pp. 263-282, 2008.

[27] I. S. Jesus, J. A. T. Machado, and R. S. Barbosa, "Control of a heat diffusion system through a fractional order nonlinear algorithm," Computers and Mathematics with Applications, vol. 59, no. 5, pp. 1687-1694, 2010

[28] T. J. Anastasio, "The fractional-order dynamics of brainstem vestibulo-oculomotor neurons," Biological Cybernetics, vol. 72, no. 1, pp. 69-79, 1994.

[29] M. D. Ortigueira and J. A. T. Machado, "Fractional calculus applications in signals and systems," Signal Processing, vol. 86, no. 10, pp. 2503-2504, 2006.

[30] R. L. Magin, Fractional Calculus in Bioengineering, Begll House, Redding, Conn, USA, 2006.

[31] V. Daftardar-Gejji and A. Babakhani, "Analysis of a system of fractional differential equations," Journal of Mathematical Analysis and Applications, vol. 293, no. 2, pp. 511-522, 2004.

[32] V. Daftardar-Gejji and H. Jafari, "Analysis of a system of nonautonomous fractional differential equations involving Caputo derivatives," Journal of Mathematical Analysis and Applications, vol. 328, no. 2, pp. 1026-1033, 2007. 
[33] W. H. Deng and C. P. Li, "Chaos synchronization of the fractional Lü system," Physica A, vol. 353, no. 1-4, pp. 61-72, 2005.

[34] W. Deng and C. Li, "Synchronization of chaotic fractional Chen system," Journal of the Physical Society of Japan, vol. 74, no. 6, pp. 1645-1648, 2005.

[35] C. Li and W. Deng, "Chaos synchronization of fractional-order differential systems," International Journal of Modern Physics B, vol. 20, no. 7, pp. 791-803, 2006.

[36] T. Zhou and C. Li, "Synchronization in fractional-order differential systems," Physica D, vol. 212, no. 1-2, pp. 111-125, 2005.

[37] C. P. Li, W. H. Deng, and D. Xu, "Chaos synchronization of the Chua system with a fractional order," Physica A, vol. 360, no. 2, pp. 171-185, 2006.

[38] J. Wang, X. Xiong, and Y. Zhang, "Extending synchronization scheme to chaotic fractional-order Chen systems," Physica A, vol. 370, no. 2, pp. 279-285, 2006.

[39] J. Wang and Y. Zhang, "Designing synchronization schemes for chaotic fractional-order unified systems," Chaos, Solitons and Fractals, vol. 30, no. 5, pp. 1265-1272, 2006.

[40] X. Y. Wang and Y. He, "Projective synchronization of fractional order chaotic system based on linear separation," Physics Letters, Section A, vol. 372, no. 4, pp. 435-441, 2008.

[41] Y. Yu and H. X. Li, "The synchronization of fractional-order Rössler hyperchaotic systems," Physica A, vol. 387, no. 5-6, pp. 1393-1403, 2008.

[42] M. S. Tavazoei and M. Haeri, "Synchronization of chaotic fractional-order systems via active sliding mode controller," Physica A, vol. 387, no. 1, pp. 57-70, 2008.

[43] A. E. Matouk, "Chaos synchronization between two different fractional systems of Lorenz family," Mathematical Problems in Engineering, vol. 2009, Article ID 572724, 11 pages, 2009.

[44] J. Hu, Y. Han, and L. Zhao, "Synchronizing chaotic systems using control based on a special matrix structure and extending to fractional chaotic systems," Communications in Nonlinear Science and Numerical Simulation, vol. 15, no. 1, pp. 115-123, 2010.

[45] S. Bhalekar and V. Daftardar-Gejji, "Synchronization of different fractional order chaotic systems using active control," Communications in Nonlinear Science and Numerical Simulation, vol. 15, no. 11, pp. 3536-3546, 2010.

[46] Y. Luchko and R. Gorenflo, "An operational method for solving fractional differential equations with the Caputo derivatives," Acta Mathematica Vietnamica, vol. 24, pp. 207-233, 1999.

[47] K. Diethelm, N. J. Ford, and A. D. Freed, "A predictor-corrector approach for the numerical solution of fractional differential equations," Nonlinear Dynamics, vol. 29, no. 1-4, pp. 3-22, 2002.

[48] K. Diethelm, "An algorithm for the numerical solution of differential equations of fractional order," Electronic Transactions on Numerical Analysis, vol. 5, pp. 1-6, 1997.

[49] K. Diethelm and N. J. Ford, "Analysis of fractional differential equations," Journal of Mathematical Analysis and Applications, vol. 265, no. 2, pp. 229-248, 2002.

[50] I. Grigorenko and E. Grigorenko, "Chaotic dynamics of the fractional Lorenz system," Physical Review Letters, vol. 91, no. 3, Article ID 034101, 4 pages, 2003.

[51] X. J. Wu and S. L. Shen, "Chaos in the fractional-order Lorenz system," International Journal of Computer Mathematics, vol. 86, no. 7, pp. 1274-1282, 2009.

[52] W. C. Chen, "Nonlinear dynamics and chaos in a fractional-order financial system," Chaos, Solitons and Fractals, vol. 36, no. 5, pp. 1305-1314, 2008.

[53] C. Li and G. Peng, "Chaos in Chen's system with a fractional order," Chaos, Solitons and Fractals, vol. 22, no. 2, pp. 443-450, 2004.

[54] J. G. Lü, "Chaotic dynamics of the fractional-order Lü system and its synchronization," Physics Letters, Section A, vol. 354, no. 4, pp. 305-311, 2006.

[55] D. Matignon, "Stability results for fractional differential equations with applications to control processing," in Proceedings of the IEEE-SMC Computational Engineering in Systems and Application multiconference (IMACS '96), vol. 2, pp. 963-968, Lille, France, July 1996.

[56] M.S. Tavazoei and M. Haeri, "Chaotic attractors in incommensurate fractional order systems," Physica D, vol. 237, no. 20, pp. 2628-2637, 2008.

[57] M. S. Tavazoei and M. Haeri, "A necessary condition for double scroll attractor existence in fractionalorder systems," Physics Letters, Section A, vol. 367, no. 1-2, pp. 102-113, 2007. 


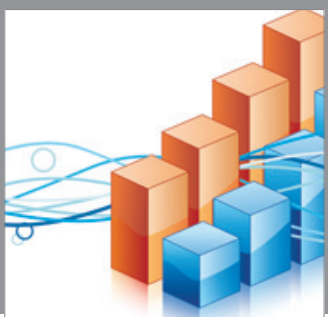

Advances in

Operations Research

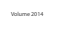

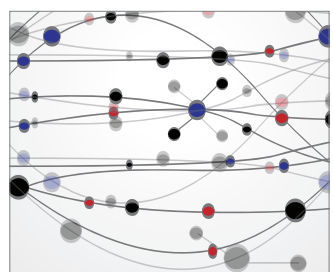

\section{The Scientific} World Journal
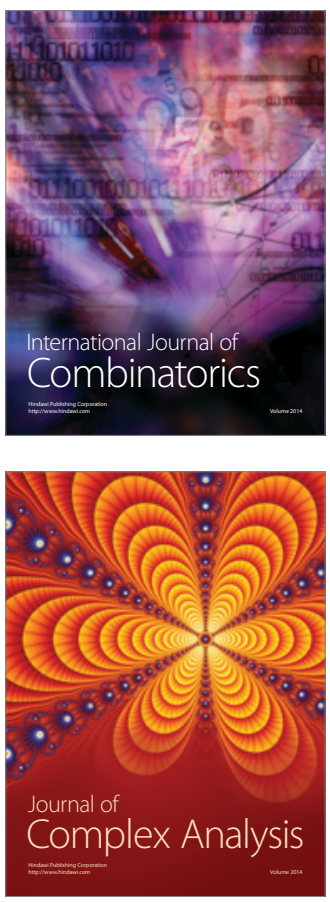

International Journal of

Mathematics and

Mathematical

Sciences
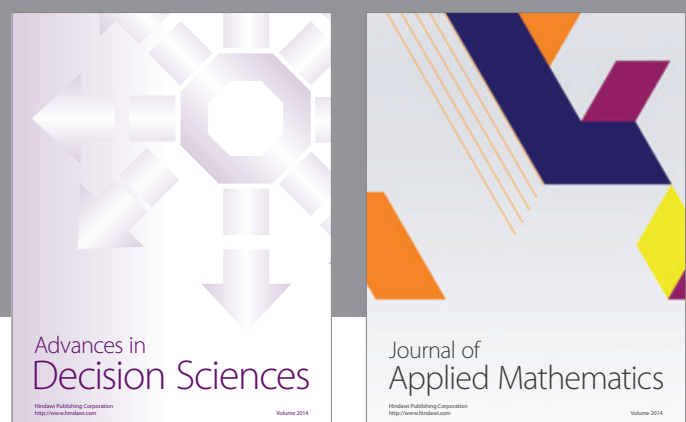

Journal of

Applied Mathematics
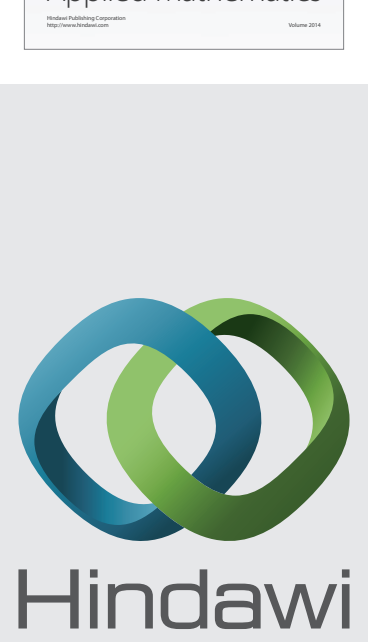

Submit your manuscripts at http://www.hindawi.com
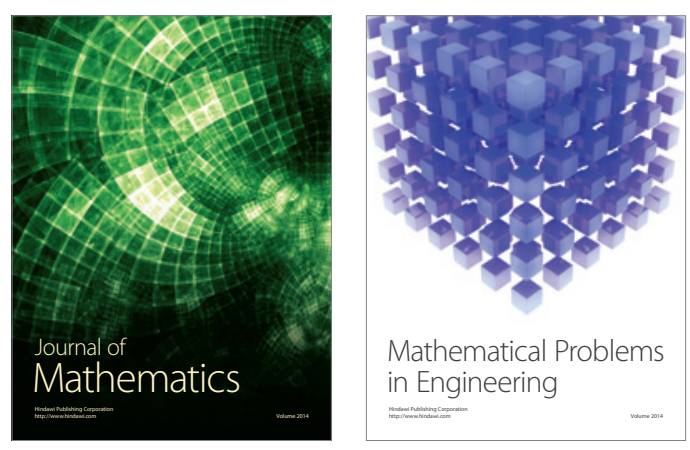

Mathematical Problems in Engineering
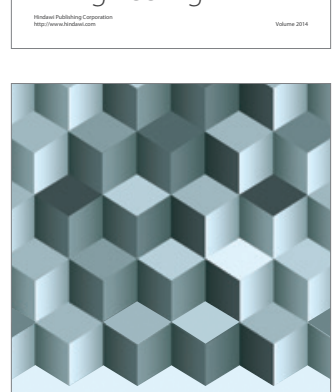

Journal of

Function Spaces
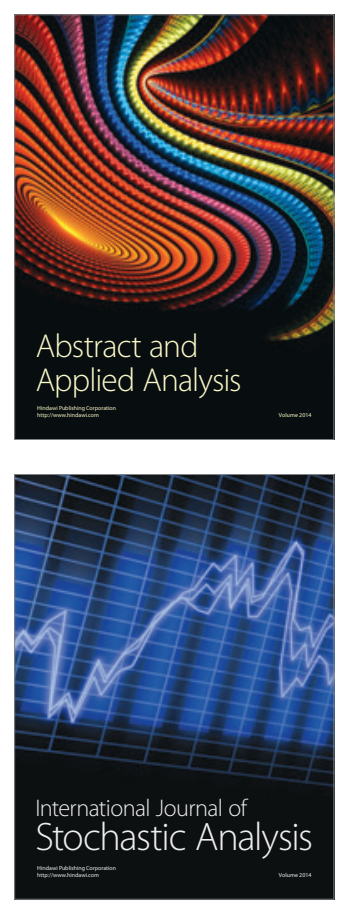

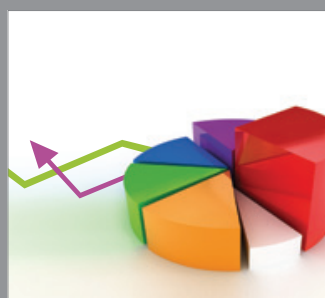

ournal of

Probability and Statistics

Promensencen
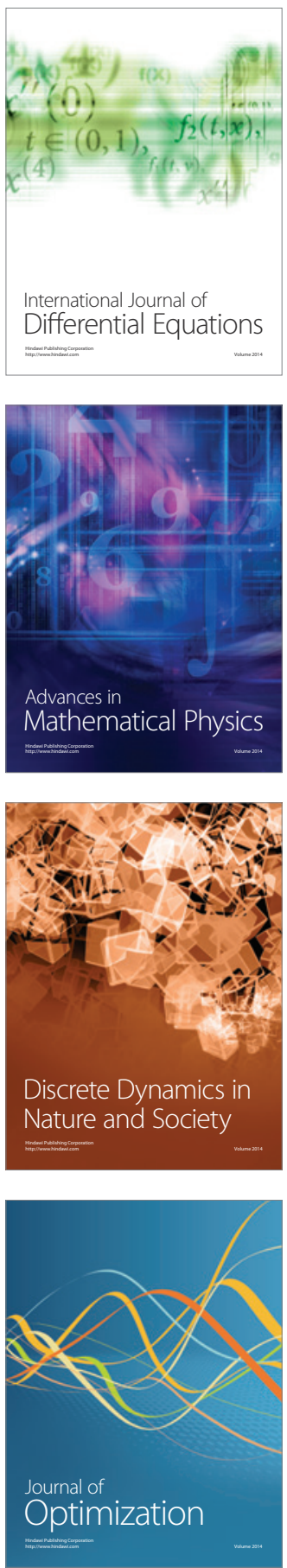